\title{
Micro-arcsecond astrometry with the VLBA
}

\author{
M. J. Reid \\ Harvard-Smithsonian CfA, Cambridge, MA, 02138, USA \\ email: reid@cfa.harvard.edu
}

\begin{abstract}
The VLBA is now achieving parallaxes and proper motions with accuracies approaching the micro-arcsecond domain. The apparent proper motion of Sgr A*, which reflects the orbit of the Sun around the Galactic center, has been measured with high accuracy. This measurement strongly constrains $\Theta_{0} / \mathrm{R}_{0}$ and offers a dynamical definition of the Galactic plane with Sgr A*at its origin. The intrinsic motion of Sgr A*is very small and comparable to that expected for a supermassive black hole. Trigonometric parallaxes and proper motions for a number of massive star forming regions (MSFRs) have now been measured. For almost all cases, kinematic distances exceed the true distances, suggesting that the Galactic parameters, $R_{0}$ and $\Theta_{0}$, are inaccurate. Solutions for the Solar Motion are in general agreement with those obtained from Hipparcos data, except that MSFRs appear to be rotating slower than the Galaxy. Finally, the VLBA has been used to measure extragalactic proper motions and to map masers in distant AGN accretion disks, which will yield direct estimates of $\mathrm{H}_{0}$.
\end{abstract}

Keywords. Galactic: structure, stars: kinematics, black hole physics, masers, astrometry, instrumentation: interferometers, stars: fundamental parameters, cosmological parameters

\section{The proper motion of Sgr A*}

Since 1995, the VLBA has been used to measure the proper motion of the compact radio source Sgr A* relative to background quasars. The apparent motion comes predominantly from observing in the solar system, which orbits the Galactic center. With single epoch positional accuracies now better than about 0.1 mas, the VLBA is able to detect the effects of this $225 \mathrm{My}$ orbit in less than one month's observing!

Were Sgr A* a stellar-mass object, for example an X-ray binary including a neutron star or black hole, it would be moving at thousands of $\mathrm{km} \mathrm{s}^{-1}$, as stars close to the position of Sgr A* are observed to do. However, for a supermassive black hole (SMBH) at the Galactic center, one expects a very small motion of $\sim 0.3 \mathrm{~km} \mathrm{~s}^{-1}$ owing to small random perturbations from the $\sim 10^{6}$ stars (and perhaps upwards of $10^{4}$ stellar remnants) within Sgr A*'s gravitational sphere of influence. With 8 years of VLBA data, Reid \& Brunthaler (2004) find that the true motion of Sgr A* in the direction perpendicular to the Galactic plane is indeed very small: $-0.4 \pm 0.9 \mathrm{~km} \mathrm{~s}^{-1}$. Preliminary analysis of data taken in 2007, shown in Fig. 1, extends the time baseline to 11 years and should reduce the uncertainty in Sgr A*'s motion below $\pm 0.7 \mathrm{~km} \mathrm{~s}^{-1}$.

Infrared observations of stars in elliptical orbits about the position of Sgr A* by Schödel et al. (2002) and Ghez et al. (2005) demonstrate conclusively that there is an unseen mass of $\sim 4 \times 10^{6} \mathrm{M}_{\odot}$ near the position of Sgr $\mathrm{A}^{*}$. The existence of this dark mass, coupled with the upper limit for the intrinsic motion of Sgr $A^{*}$, implies a lower limit for Sgr A*'s mass of $\sim 4 \times 10^{5} \mathrm{M}_{\odot}$. Combining this mass lower limit with an upper limit of $\approx 0.5 \mathrm{AU}$ for the intrinsic (non-scatter broadened) size of Sgr A* (see Bower et al. (2004) and references therein), results in a mass density $>7 \times 10^{21} \mathrm{M}_{\odot} \mathrm{pc}^{-3}$. This lower limit is within only about two orders of magnitude of a $\mathrm{SMBH}$ within the inner-most stable orbit 


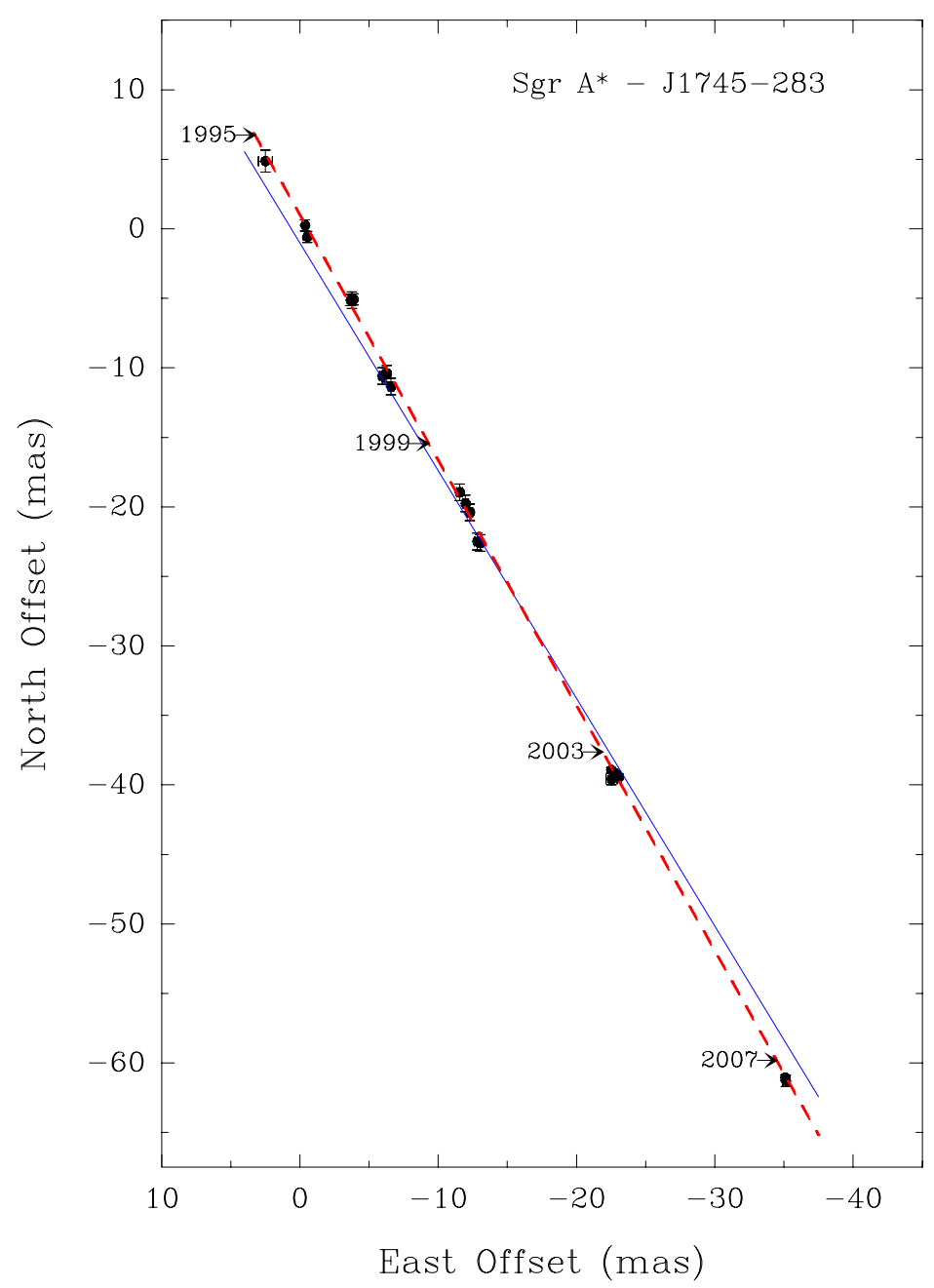

Figure 1. Apparent proper motion of Sgr A* relative to the extragalactic source J1745-283 from Reid \& Brunthaler (in preparation). Time is indicated at the beginning of every fourth year. The apparent motion is caused predominantly by our moving vantage point in the solar system as it orbits about the Galactic center every $\approx 225 \mathrm{My}$. The red dashed line is the best fit proper motion and the blue solid line is the orientation of the IAU Galactic plane. The difference between these lines can be accounted for by the motion of the Sun toward the north Galactic pole, implying that $\mathrm{Sgr} \mathrm{A}$ * is motionless to within about $1 \mathrm{~km} \mathrm{~s}^{-1}$ in this direction.

for a Schwarzschild black hole. This provides overwhelming evidence that Sgr $\mathrm{A}^{*}$ is a SMBH.

Recognizing that Sgr A* is a SMBH that anchors the dynamical center of the Galaxy and that its apparent proper motion is dominated by the orbit of the Sun about the Galactic center, one could use this to provide a dynamical definition of the plane of the Galaxy. The current IAU definition of the Galactic plane is based primarily on the distribution of HI, with the Sun arbitrarily defining zero latitude. The Sun is now known to be $\sim 10-20 \mathrm{pc}$ above the IAU plane and $\operatorname{Sgr} \mathrm{A}^{*}$ is $\approx 6$ pc below the plane. A better definition of the Galactic plane could come from the direction of the apparent proper motion of Sgr A* (corrected for the small, well measured, peculiar motion of the 

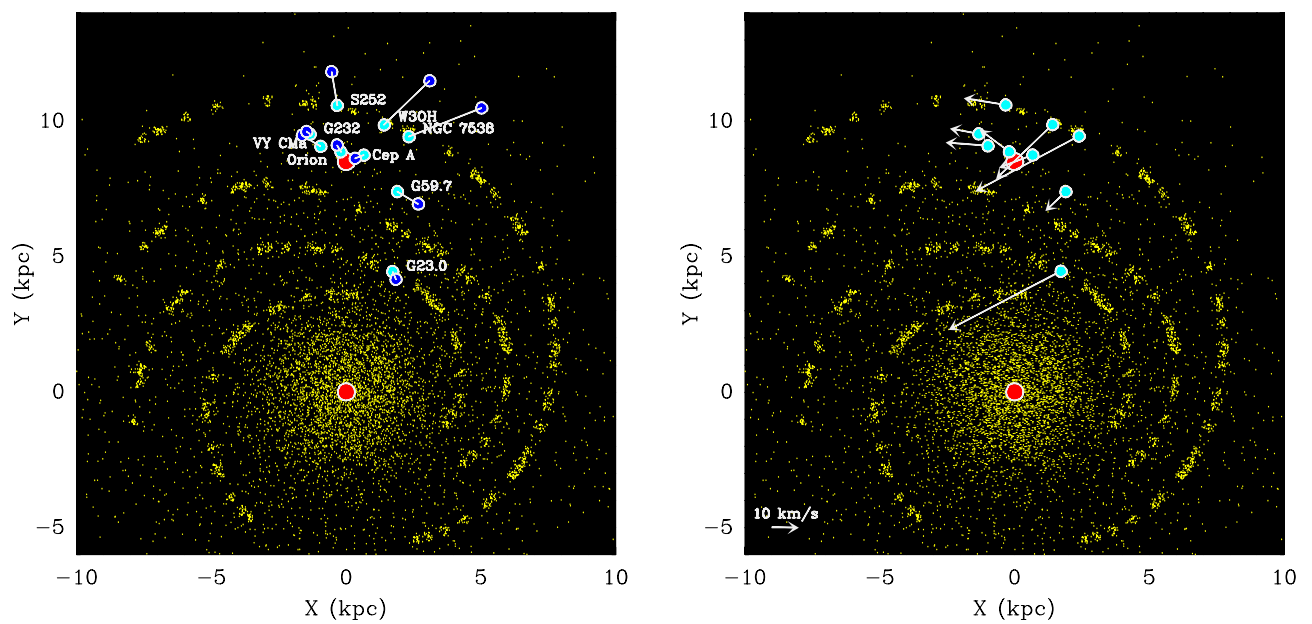

Figure 2. Left panel: Comparison of trigonometric parallaxes (light cyan circles) with kinematic distances (dark blue circles), assuming $R_{0}=8.5 \mathrm{kpc}$ and a flat rotation curve with $\Theta_{0}=220 \mathrm{~km}$ $\mathrm{s}^{-1}$. For all sources, except Cep A, the kinematic distances are larger than the trigonometric parallaxes. The background is a schematic model of the Milky Way with spiral arms from Taylor \& Cordes (1993). The position of the Sun $(0,8)$ and Sgr A* $(0,0) \mathrm{kpc}$ are also indicated. Right panel: Peculiar motions of MSFRs after removing a standard model of Galactic rotation $\left(R_{0}=8.5 \mathrm{kpc}\right.$ and a flat rotation curve with $\left.\Theta_{0}=220 \mathrm{~km} \mathrm{~s}^{-1}\right)$ and Solar Motion parameters from Dehnen \& Binney (1998).

Sun perpendicular to the plane), with Sgr A*'s position defining the zero of Galactic longitude and latitude. Of course, as the Sun orbits the Galactic center, the position of Sgr $A^{*}$ changes by about 6 mas $\mathrm{y}^{-1}$; thus the zero of Galactic longitude would become a function of time.

\section{Parallax and proper motions of star forming regions}

An international team, including A. Brunthaler, K. Menten, L. Moscadelli, M. Reid, Y. Xu and X.-W. Zheng are conducting a large program with the VLBA to map the spiral structure of the Milky Way and to define its rotational kinematics and mass profile. This involves measuring trigonometric parallaxes and proper motions to methanol (and some other) masers in massive star forming regions. We are observing primarily in the 12.2 $\mathrm{GHz}$ methanol transition, since it falls in one of the VLBA bands.

Observations for the first group of masers are complete, and preliminary parallaxes for many sources are shown in Fig. 2. We have accurate distances to massive star forming regions in several spiral arms. All sources, with the possible exception of G59.7+0.1, lie close to these spiral arms. With more measurements we plan to map the locations of the major spiral arms of the Galaxy. In Fig. 2, we also show kinematic distances. As Xu et al. (2006) found for W3OH, the Perseus arm sources S252 and NGC 7538 also have a large kinematic anomalies, with kinematic distances roughly double their trigonometric parallax distances.

Almost all sources appear to have kinematic distances that are greater than trigonometric parallax distances. Decreasing $R_{0}$ and/or increasing $\Theta_{0}$, from the adopted IAU values, would reduce the kinematic distances and bring them into better agreement with the trigonometric parallaxes. We note that the proper motion of Sgr A* by Reid \& Brunthaler (2004), after a small correction for Solar Motion, implies $\Theta_{0} / R_{0}=29.45 \pm 0.15 \mathrm{~km}$ 
$\mathrm{s}^{-1} \mathrm{kpc}^{-1}$. If $R_{0}=8.0 \mathrm{kpc}$ (Reid 1993), this requires $\Theta_{0}=236 \mathrm{~km} \mathrm{~s}^{-1}$; using these Galactic parameters would reduce most kinematic distances by roughly $10-15 \%$.

Parallax observations require solving for proper motions and, with current VLBA accuracies, proper motions with $\sim 1 \mathrm{~km} \mathrm{~s}^{-1}$ are often achieved. Proper motions, coupled with radial velocities obtained from Doppler shifts of maser or thermally excited lines, yield full space velocities. The space velocities of the MSFRs generally indicate small motions out of the plane of the Galaxy, as expected for MSFRs. However, the components of the space velocities in the plane of the Galaxy reveal a surprising result.

After removing the effects of the rotation of the Galaxy, we plot the residual motions of the MSFRs in Fig. 2 superposed on a "plan view" of the Milky Way. Nearly all sources show large residual motions, notably counter to Galactic rotation. One possibility explanation for these large residuals is that the Galactic rotation model, based on IAU standard parameters for $\mathrm{R}_{0}$ and $\Theta_{0}$, needs modification. Indeed, decreasing $\mathrm{R}_{0}$ and increasing $\Theta_{0}$, can reduce the residual motions somewhat. However, the residual motions can only be reduced to $\approx 5 \mathrm{~km} \mathrm{~s}^{-1}$ in magnitude by adopting a Solar Motion correction that is markedly different from those determined from Hipparcos data.

Figure 3 shows the Hipparcos data used by Dehnen \& Binney (1998) to determine the three components of the Solar Motion. Also plotted are the Solar Motion components required to remove the large systematic residuals for the MSFRs with VLBA parallaxes and proper motions. The components of the Solar Motion in the direction of the Galactic center (U) and toward the north Galactic pole (W) are conceptually simple to measure and there is excellent agreement between the Hipparcos and VLBA results. Also, the proper motion of Sgr A*, assuming it is nearly motionless at the Galactic center, provides a direct estimate of $\mathrm{W}$, which is in agreement with other methods.

However, the VLBA results require the component of Solar Motion in the direction of Galactic rotation (V) to be about $18 \mathrm{~km} \mathrm{~s}^{-1}$. This should be compared with the Hipparcos value of about $5 \mathrm{~km} \mathrm{~s}^{-1}$, when the "asymmetrical drift" is extrapolated to zero stellar dispersion (ie, the dynamical Solar Motion; see Dehnen \& Binney (1998) for a more complete discussion). The VLBA determined value for $\mathrm{V}$ is about $13 \mathrm{~km} \mathrm{~s}^{-1}$ greater than the dynamical Solar Motion. This suggests that MSFRs as a group rotate $13 \mathrm{~km} \mathrm{~s}^{-1}$ slower than the Galaxy spins. This result is fairly insensitive to the values adopted for $\mathrm{R}_{0}$ and $\Theta_{0}$. A possible explanation for this finding is that MSFRs are born near apocenter in slightly elliptical Galactic orbits, perhaps owing to the effects of spiral density wave shocks. Such shocks could compress gas clouds and induce star formation and remove angular momentum from originally circular Galactic orbits.

\section{Extra-galactic VLBA astrometry}

Even with near micro-arcsecond astrometry, one cannot measure parallaxes to other galaxies with high accuracy. However, proper motion accuracy increases with the spanned observing time, $T$, as $T^{-3 / 2}$. An international team including A. Brunthaler, H. Falcke and M. Reid have succeeded in measuring both the internal angular rotation and the proper motion of M33 with the VLBA. These observations constrain the dark matter in the Local Group. For more details, see the paper by A. Brunthaler in these proceedings.

One of the most interesting problems in contemporary cosmology is to determine the equation of state $(w)$ of dark energy. Observations of primordial fluctuations in the Cosmic Microwave Background, supplemented with data from supernovae, gravitational lensing and galaxy distributions, suggest $w$ near unity. However, this assumes either a flat universe or a value for the Hubble Constant $\left(\mathrm{H}_{0}\right)$. An independent and accurate determination of $\mathrm{H}_{0}$ is crucial for a more accurate measure of $w$. 

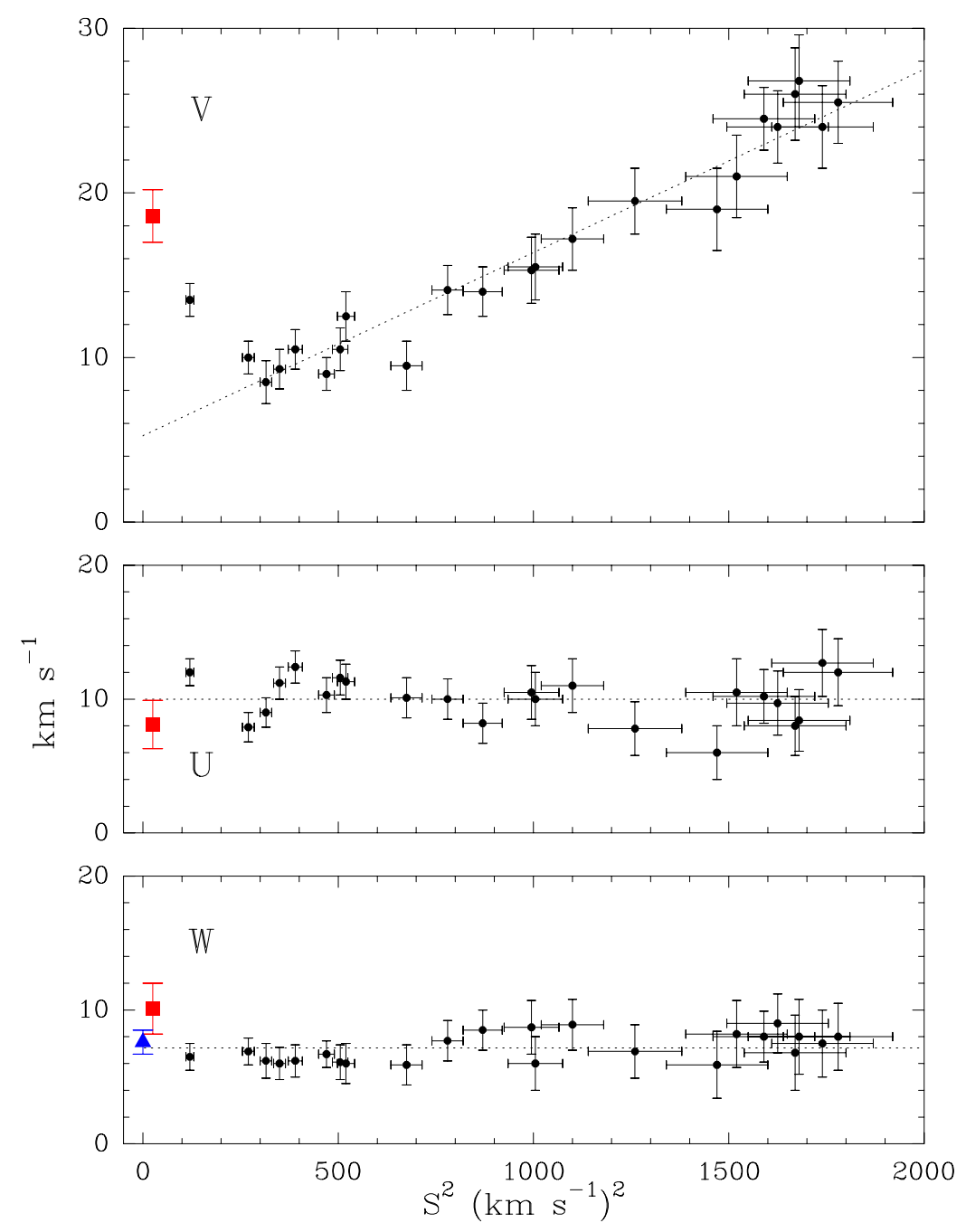

Figure 3. Solar Motion estimates. Plotted are the three components of Solar Motion (U toward the Galactic center, V in the direction of Galactic rotation; W toward the north Galactic pole) versus the dispersion of groups of stars used to determine the Solar Motion. Black points are from Hipparcos data by Dehnen \& Binney (1998); red squares are from MSFR parallaxes and proper motions; the blue triangle is from the proper motion of Sgr A*. The well-known "asymmetrical drift" (dotted line) is seen in the V plot of the Hipparcos data; extrapolating to zero stellar dispersion defines the dynamical Solar Motion.

The Seyfert 2 galaxy NGC 4258 has a sub-pc scale accretion disk in its nucleus that can be traced by VLBI imaging of its $\mathrm{H}_{2} \mathrm{O}$ maser emission. Herrnstein et al. (1999) determined a highly accurate geometric distance of $7.2 \mathrm{Mpc}$ to this galaxy. Since the recessional velocity of NGC 4258 is only $470 \mathrm{~km} \mathrm{~s}^{-1}$, the possibility of non-Hubble flow motions of several hundred $\mathrm{km} \mathrm{s}^{-1}$ precludes a direct estimate of $\mathrm{H}_{0}$. However, NGC 4258 has been used to re-calibrate the Cepheid-based extragalactic distance scale.

If galaxies like NGC 4258, but more distant and into the "Hubble flow" can be imaged with VLBI observations, then direct determinations of $\mathrm{H}_{0}$ are possible. The Water Maser Cosmology Project (WMCP) is an international collaboration, including J. Braatz, 


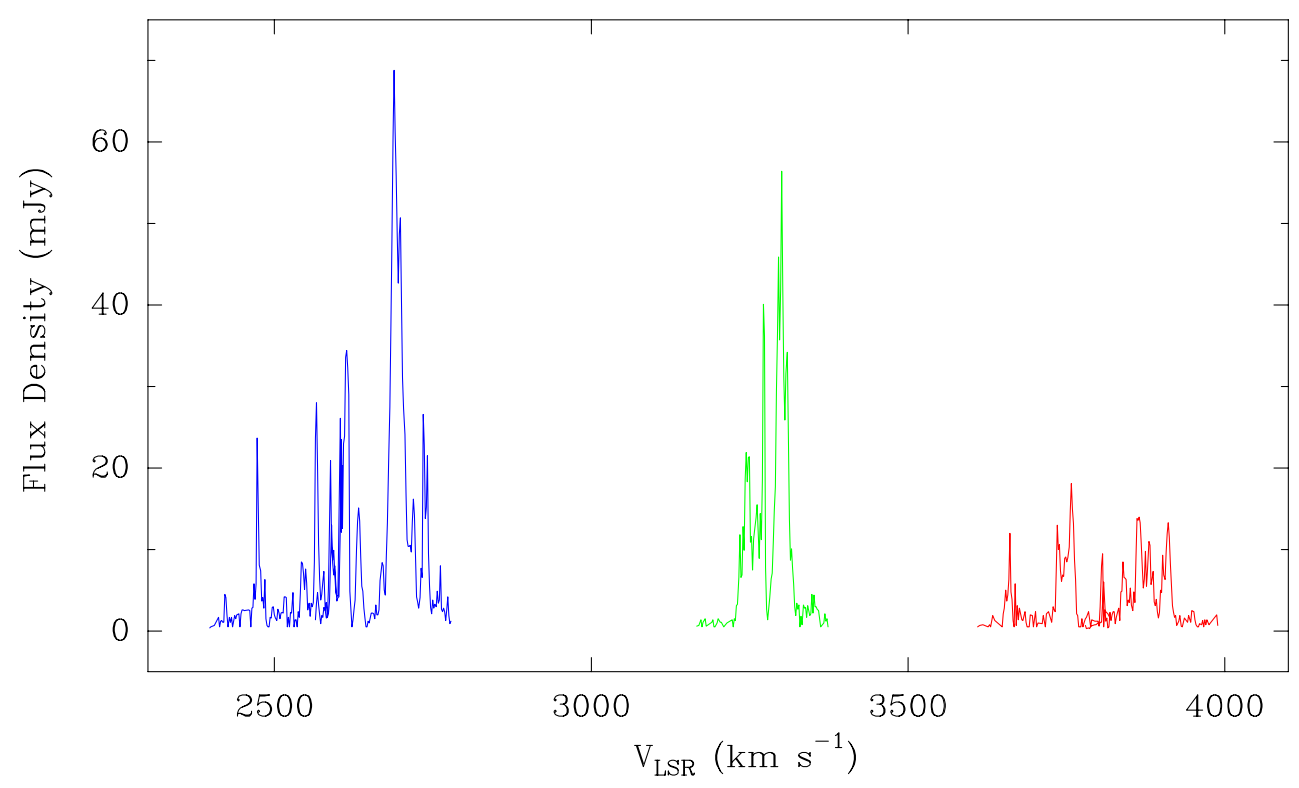

Figure 4. Interferometric spectrum of the $\mathrm{H}_{2} \mathrm{O}$ masers towards UGC 3789 from a map made with VLBA plus GBT data. Note the similarities with the spectrum of NGC 4258: blue- and red-shifted high-velocity emission complexes that straddle the systemic emission complex centered near $3300 \mathrm{~km} \mathrm{~s}^{-1}$.

J. Condon, L. Greenhill, C. Henkel, K.-Y. Lo, and M. Reid, that seeks to do this. Its goal is to measure $\mathrm{H}_{0}$ directly with an accuracy of better than $3 \%$. The WMCP uses the VLBA, along with large aperture telescopes such as the GBT and the Effelsberg 100-m telescope, to both monitor and map the $\mathrm{H}_{2} \mathrm{O}$ maser emission from NGC 4258-like accretion disks in AGN.

One of the most promising candidates for distance measurement is UGC 3789, which with a recessional velocity of near $3300 \mathrm{~km} \mathrm{~s}^{-1}$ is into the Hubble flow. This source was discovered by J. Braatz using the GBT. The $\mathrm{H}_{2} \mathrm{O}$ masers have been imaged with the VLBA/GBT and an interferometer spectrum is presented in Fig. 4. This spectrum displays the characteristics of a sub-pc scale accretion disk surrounding a SMBH: systemic velocity components flanked by high-velocity components separated by up to $\pm 750 \mathrm{~km}$ $\mathrm{s}^{-1}$.

A map of the $\mathrm{H}_{2} \mathrm{O}$ masers in UGC 3789 with positional accuracies approaching $\sim 5 \mu$ as is shown in the left panel of Fig. 5. As for NGC 4258, the maser spots fall in a linear pattern with the blue- and red-shifted high velocity components straddling the systemic velocity components, indicative of an edge-on disk. The angular extent of the detected spots is about 1.5 mas, about a factor of 10 smaller than for NGC 4258 and consistent with UGC 3789's much greater distance.

The right panel of Fig. 5 shows a position-velocity plot constructed along the position angle of the linear pattern of spots. One can clearly see a Keplerian rotation curve for the high velocity components, indicating a rotation speed of $\approx 600 \mathrm{~km} \mathrm{~s}^{-1}$ at an angular radius of $\approx 0.5$ mas. This implies a central $\mathrm{SMBH}$ of $10^{7}(D / 50 \mathrm{Mpc}) \mathrm{M}_{\odot}$, where $D$ is the distance.

The distance to such a source with an edge-on rotating disk is given by $D=V^{2} / A \theta$, where $V$ and $A$ are the rotational velocity and centripetal acceleration at an angular 

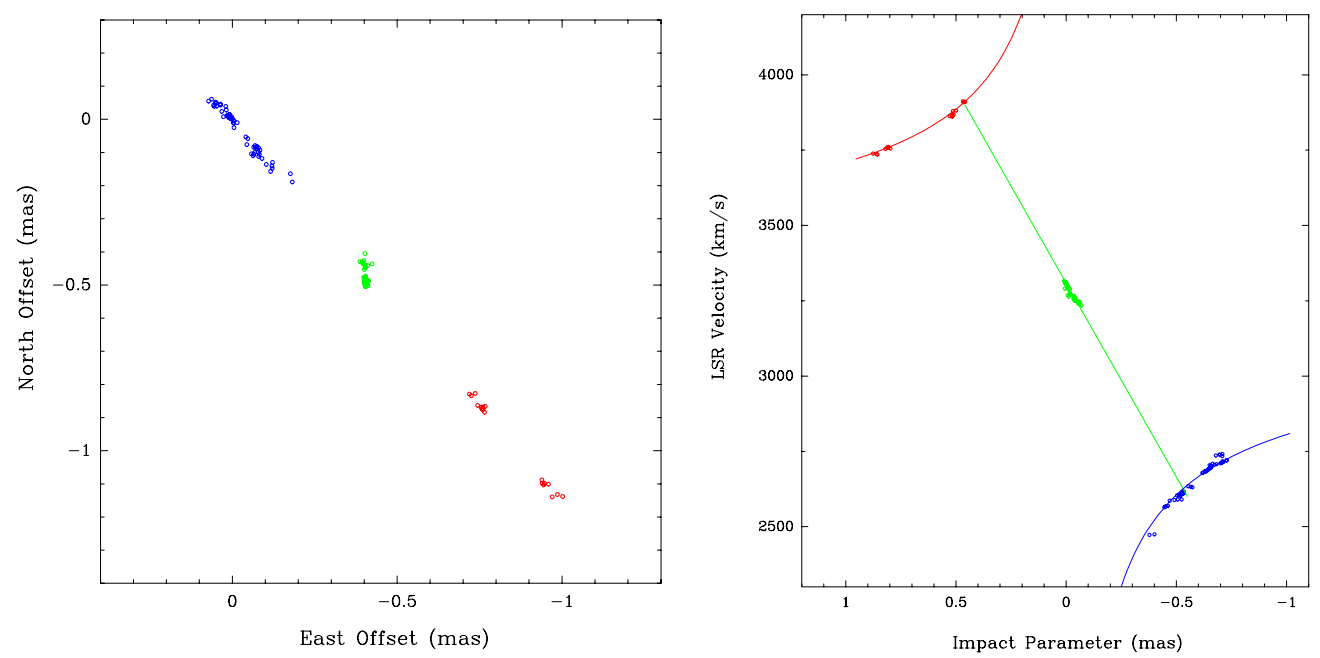

Figure 5. Map (left panel) and position-velocity plot (right panel) of $\mathrm{H}_{2} \mathrm{O}$ masers toward UGC 3789. In the map the blue-shifted (red-shifted) high-velocity components are offset to the East (West) of the systemic masers near $(-0.4,-0.5)$ mas. In the position-velocity plot, the blue- and red-shifted components indicate a Keplerian rotation.

radius $\theta$. Centripetal accelerations can be measured from the drift over time of Doppler shifts of maser features. Preliminary measurement of $A$, based on one year's monitoring with the GBT, gives distances consistent with $\mathrm{H}_{0}$ near $70 \mathrm{~km} \mathrm{~s}^{-1} \mathrm{Mpc}^{-1}$ and an anticipated uncertainty of about $\pm 10 \%$. If achieved, this would give an independent value of $\mathrm{H}_{0}$ with an accuracy comparable to that of the Hubble Key Project (Freedman et al.2001).

\section{References}

Bower, G. C. Falcke, H. Herrnstein, R. M, Zhao, J.-H., Goss, W. M., \& Backer, D. C. 2004, Science 304, 704

Dehnen, W. \& Binney, J. J. 1998, MNRAS, 298, 387

Freedman et al. 2001, ApJ, 553, 47

Ghez et al. 2005, ApJ, 620, 744

Herrnstein et al. 1999, Nature, 400, 539

Reid, M. J. \& Brunthaler, A. 2004, ApJ 616, 872

Schödel et al. 2002, Nature, 419, 694

Taylor, J. H. \& Cordes, J. M. 1993, ApJ 411, 674

Xu, Y., Reid, M. J., Zheng, X. W., \& Menten, K. M. 2006, Science, 311, 54 